\title{
EFECTOS DE LA PRIMERA VISITA DE CONSEJO GENÉTICO SOBRE LA PERCEPCIÓN DE RIESGO Y EL MALESTAR EMOCIONAL
}

\section{PERCEIVED RISK AND EMOTIONAL DISTRESS AFTER THE FIRST VISIT AT A GENETIC COUNSELING UNIT}

\author{
Melinda González', Ignacio Blanco², Tomás Blasco' \\ 1 Unitat de Psicologia Bàsica. Universitat Autònoma de Barcelona. \\ 2 Unidad de Consejo Genético. Hospital Duran i Reynals, Institut Català d'Oncologia.
}

Resumen

Objetivo: Analizar los efectos que provoca la primera visita de consejo genético para cáncer hereditario sobre la percepción de riesgo, y el malestar emocional en pacientes que acuden por primera vez a la Unidad de Consejo Genético (UCG) por historia familiar de cáncer de mama, ovario o colon hereditario.

Método: Se evaluó la percepción de riesgo de padecer cáncer (al año, a 10 años, y a lo largo de toda la vida), y la intensidad del malestar emocional (evaluada como la ansiedad, tristeza y preocupación por la salud experimentadas durante la última semana) en tres ocasiones: inmediatamente antes de la primera visita de consejo genético (CG), inmediatamente después de la misma, y al cabo de 2-3 semanas.

Resultados: El malestar emocional previo a la visita se producía en aproximadamente un $30 \%$ de los pacientes, siendo mayor en las personas que no realizaban actividad laboral. Si bien la visita de CG reducía el malestar emocional en muchos casos, ocurría lo contrario en algunos pacientes inmediatamente después de la misma (un $34 \%$ se sentía más ansioso, un $32 \%$ se sentía más triste, y un $23 \%$ se sentía más preocupado), y ello guardaba relación con la preocupación por los hijos (tanto si se tenían como si se pretendía tenerlos en el futuro). No obstante, este malestar se reducía en las 2-3

\section{Abstract}

Objective: To analyze how perceived risk and emotional distress were affected by the first visit to a Genetic Counseling Unit in patients with a family history of hereditarian breast cancer, ovarian cancer, or colorectal cancer.

Methods: Risk perception of suffering cancer at $\mathbf{1}$ year, at $\mathbf{1 0}$ years, and across the life span, as well as emotional distress (assessed as sadness, anxiety and worries during the last week) was assessed in three moments: before the first visit to the Genetic Counseling Unit, immediately after finishing the visit, and 2-3 weeks after the first visit.

Results: Emotional distress prior the first visit was observed in $30 \%$ of patients, with higher levels in patients without job. The first visit decreased emotional distress in most cases. However, $34 \%$ of patients stated that they were more anxious after the visit, $32 \%$ of patients become sadder, and $23 \%$ of patients were more worried. Patients who expressed increased levels of emotional distress after the visit were worried about their sons (or sons they wanted to have in the future). In spite of that, this emotional distress decreased at 2-3 weeks. The first visit to a Genetic Counseling Unit decreased risk perception of having cancer across the life span $(p<.01)$, and there were not differences between the three types of cancer.

\section{Correspondencia:}

Tomás Blasco.

Departament de Psicologia Bàsica, evolutiva i de l'Educació.

Edifici $B$.

Universitat Autònoma de Barcelona.

08193 Bellaterra (Barcelona) SPAIN.

E-mail: Tomas.Blasco@uab.es 
semanas posteriores. La visita de CG reducía la percepción de riesgo de padecer cáncer a lo largo de toda la vida $(p<.01)$, no existiendo diferencias en función del tipo de cáncer.

Conclusiones: Esta primera visita reduce la sobreestimación del riesgo de padecer cáncer que los pacientes tienen antes de la misma, pero, en aproximadamente un tercio de los mismos, no reduce los niveles de malestar emocional, observándose, incluso, un incremento de éste que va disminuyendo durante las semanas posteriores a la visita. Son necesarios más estudios que permitan determinar si este incremento es una consecuencia inevitable del inicio del proceso de CG, o si puede reducirse modificando algún aspecto del protocolo que se aplica en la primera visita.

Palabras clave: Consejo genético, percepción de riesgo, malestar emocional.
Conclusions: First visit to a Genetic Counseling Unit decreases overestimation of risk perception of having cancer. Approximately one third of patients increased their emotional distress after the visit. Most studies are needed in order to clarify whether this increase in emotional distress is unavoidable for some patients at the beginning of the genetic counseling process, or it can be reduced if some features of the protocol applied during the visit are changed.

Key Words: Genetic Counselling, Risk perception, Emotional Distress.

\section{INTRODUCCIÓN}

Aun cuando pueda considerarse que el consejo genético en cáncer se inicia con la primera visita a la unidad correspondiente, lo cierto es que para las personas que acuden el proceso ya ha provocado efectos que, en muchos casos, tienen connotaciones negativas como preocupación (por uno mismo y por los familiares), incertidumbre, o sensación de riesgo. Al llegar a la primera visita existen diferentes perfiles de pacientes dependiendo del grado de conocimiento que las personas puedan tener sobre el cáncer y el consejo genético, pero se ha detectado que existe una importante falta de información y una sobrestimación del riesgo ${ }^{(1-2)}$,así como, en un porcentaje de casos situado alrededor del $10 \%$, una sintomatología psiquiátrica como reacción al hecho de tener que iniciar consejo genético ${ }^{(3)}$. Asimismo, niveles elevados de ansiedad (en torno al 40\%$45 \%$ ) y de depresión (en torno al 25\%$30 \%$ ) se han encontrado también en otros estudios $^{(4-5)}$. Este malestar emocional puede venir modulado, en buena medida, por el tiempo de espera transcurrido desde la indicación de iniciar el proceso de consejo genético por parte del médico de referencia de la familia, hasta la primera visita en la unidad donde será evaluado del consejo genético propiamente dicho ${ }^{(6)}$.

Bajo estas consideraciones, queda claro que la primera visita de consejo genético juega un papel crucial, dado que no sólo tiene que informar de una situación compleja cuya comprensión es difícil para muchos pacientes $^{(7)}$, sino que debe manejar ese cuadro de malestar emocional y de incertidumbre que acabamos de describir. Así pues, la primera visita de consejo genético debe tener una estructura adecuada que permita facilitar la comprensión de la información y ajustar los niveles de percepción de riesgo, favoreciendo así el inicio y mantenimiento del proceso de consejo genético. Es, por tanto, muy importante saber qué efectos produce la primera visita de consejo genético sobre los pacientes y si efectivamente dicha visita consigue reducir o eliminar las distorsiones en la información, y ajustar los niveles de malestar emocional, así como conocer 
qué factores pueden determinar que dichos resultados se consigan en mayor o menor grado, teniendo en cuenta que la propia visita puede producir por sí misma un aumento del malestar emocional al poner de manifiesto vivencias anteriores de enfermedad en la propia persona o en sus familiares. Igualmente, la primera visita debe tener en cuenta que la realización del test genético puede generar malestar, y aun cuando dicho malestar pueda reducirse y volver a los niveles previos a la primera visita al cabo de algunos meses ${ }^{(8)}$, será más elevado en aquellos pacientes a los que se les comunica un resultado positivo de dicho test ${ }^{(9)}$.

En este contexto, el objetivo del presente trabajo es el de valorar algunos de los efectos que produce la primera visita de consejo genético en personas con historia familiar de cáncer de mama y/o ovario hereditario $(\mathrm{CMOH})$ o de cáncer de colon hereditario no polipósico (CCHNP). Concretamente, se pretende evaluar en qué medida cambian la percepción de riesgo y el malestar emocional como consecuencia de la visita en pacientes considerados como de nivel de riesgo moderado de padecer cáncer.

\section{MÉTODO}

\section{Participantes}

Los participantes en esta investigación formaban parte de un estudio más amplio realizado en la Unidad de Consejo Genético del Institut Català d'Oncología (ICO) de L'Hospitalet de Llobregat, a partir del cual se extrajo una submuestra de los pacientes que no habían padecido cáncer en ningún momento de su vida y fueron considerados pacientes de riesgo moderado. Dicha submuestra se recogió entre los meses de enero a septiembre de 2006 y estaba compuesta por un total de 43 pacientes cuyas características se indican en la Tabla 1 y que cumplían los criterios siguientes: a) Ser mayor de 18 años con historial familiar de cáncer de mama y/o ovario hereditario $(\mathrm{CMOH})$ o de cáncer de colon hereditario no polipósico (CCHNP) y acudir por primera vez a la unidad de consejo genético; b) No ser analfabetos y no tener enfermedad mental o limitación cognitiva.

\section{Material}

Evaluación inmediatamente antes de la primera visita:

La percepción de riesgo es un concepto cuya comprensión puede ser difícil para muchos pacientes. Dado que existe una correlación directa entre preocupación y nivel de riesgo percibido ${ }^{(10)}$, se consideró que la evaluación del riesgo percibido se obtenía de manera más fácil preguntando a los pacientes por su nivel de preocupación por padecer cáncer, en tres momentos diferentes: al año, a los 10 años y a lo largo de la vida. En cada caso, la persona evaluaba el grado de preocupación mediante una escala numérica en la que $0=$ Ninguna preocupación, y 10=Máxima preocupación.

Para valorar el malestar emocional se elaboraron tres ítems que preguntaban por el nivel de nerviosismo, tristeza, y preocupación que había sentido la persona en las últimas semanas. En todos los casos, se respondía con una escala categorial con los siguientes valores: "Nada", "Un poco", "Bastante", "Mucho", "Muchísimo". Para facilitar los análisis posteriores, se decidió agrupar los niveles de malestar emocional en dos categorías. Se consideró que los valores "Nada" o "Un poco" indicaban niveles de tristeza, preocupación, o nerviosismo, que no alcanzaban el malestar emocional, mientras que los valores "bastante", "mucho" o "muchísimo" sí indicaban malestar emocional. 
Tabla 1. Características de los pacientes que acuden por primera vez a la Unidad de Consejo Genético

\begin{tabular}{|c|c|c|c|}
\hline \multicolumn{4}{|c|}{ DIAGNÓSTICO POR EL CUAL ACUDEN A LA UNIDAD DE CONSEJO GENÉTICO } \\
\hline & $\mathrm{CMOH}(\%)$ & $\mathrm{CCHNP}(\%)$ & Total \\
\hline$N=43$ & $15(35)$ & $28(65)$ & 43 \\
\hline \multicolumn{4}{|l|}{ Sexo } \\
\hline Hombre & 0 & 7 & 7 \\
\hline Mujer & $15(41)$ & $21(58)$ & 36 \\
\hline \multicolumn{4}{|l|}{ Edad } \\
\hline$M(S D)$ & $39,5(14,0)$ & $44,8(10,6)$ & $43(12,0)$ \\
\hline Rango & 21-63 & 20-66 & $20-66$ \\
\hline \multicolumn{4}{|l|}{ Tener hijos } \\
\hline 0 & $6(31)$ & $13(68)$ & 19 \\
\hline 1 & $3(50)$ & $3(50)$ & 6 \\
\hline 2 & $6(35)$ & $11(65)$ & 17 \\
\hline$>2$ & 0 & 1 & 1 \\
\hline \multicolumn{4}{|l|}{ Estado civil } \\
\hline Soltero & $4(50)$ & $4(50)$ & 8 \\
\hline Casado/pareja & $10(31)$ & $22(69)$ & 32 \\
\hline Separado/divorciado & 1 & 2 & 3 \\
\hline \multicolumn{4}{|l|}{ Nivel de estudios } \\
\hline Sabe leer y escribir & $1(17)$ & $5(83)$ & 6 \\
\hline Primarios & $5(28)$ & $13(72)$ & 18 \\
\hline Secundarios & $7(53)$ & $6(57)$ & 13 \\
\hline Universitarios & $2(33)$ & $4(67)$ & 6 \\
\hline \multicolumn{4}{|l|}{ Situación laboral } \\
\hline Jornada completa & $10(53)$ & $9(47)$ & 19 \\
\hline Jornada parcial & $1(25)$ & $3(75)$ & 4 \\
\hline Sin actividad laboral & $4(2)$ & $16(8)$ & 20 \\
\hline \multicolumn{4}{|l|}{ Acude acompañado } \\
\hline Sí & $13(34)$ & $25(66)$ & 38 \\
\hline No & $2(40)$ & $3(60)$ & 5 \\
\hline
\end{tabular}

$\mathrm{CMOH}$ : Cáncer mama y/o ovario hereditario.

CCHNP: Cáncer de colon hereditario no polipósico.

Evaluación inmediatamente después de la primera visita

Justo al finalizar la visita, se preguntaba a la persona cómo se sentía en ese momento, ofreciéndosele, para responder, una escala categorial de siete puntos con los siguientes valores: "Mucho más tran- quilo que cuando Ilegué", "Bastante más tranquilo que cuando llegué", "Un poco más tranquilo que cuando Ilegué", "Confuso", "Un poco más nervioso que cuando llegué", "Bastante más nervioso que cuando llegué" y "Mucho más nervioso que cuando llegué". 
Evaluación a las 2-3 semanas después de la primera visita

Se utilizaron los mismos ítems que los empleados antes de la primera visita para valorar la percepción de riesgo y el malestar emocional.

\section{Procedimiento}

En la primera visita, mientras los pacientes y familiares estaban en la sala esperando ser recibidos por el médico responsable, la investigadora (MG) ofrecía la posibilidad de colaborar en el estudio, explicando brevemente el objetivo del mismo e indicando que únicamente deberían contestar a un mismo cuestionario que se les pasaría en dos momentos diferentes. La primera administración debía realizarse en ese momento, justo antes de la primera visita, mientras que la segunda administración la realizarían en su domicilio, entre 2 y 3 semanas después. Para ello recibirían por correo en el domicilio un ejemplar del cuestionario junto con un sobre franqueado y una carta con las instrucciones precisas. Si los pacientes aceptaban participar, se les proporcionaba un documento de consentimiento informado. Una vez firmado, se les entregaba el cuestionario con las preguntas, se resolvían las dudas respecto al mismo, y la investigadora salía de la sala de espera, ya que de esta manera se favorecía la intimidad y la libre expresión de las respuestas. El tiempo que necesitaban los pacientes para responder al cuestionario (que incluía otros ítems, además de los anteriormente descritos que son los que se utilizan para la presente investigación) fue de aproximadamente de 20 minutos.

Inmediatamente después de la visita, la investigadora preguntaba a la persona cómo se sentía en ese momento utilizando la escala descrita en el apartado "Evaluación inmediatamente después de la visita".
En el seguimiento de las devoluciones de los cuestionarios enviados al domicilio, si la investigadora no recibía el cuestionario en un plazo 2-3 semanas posteriores a la fecha prevista, realizaba una llamada telefónica para recordar la importancia que tenía para el equipo poder disponer de los datos, pues eso permitiría conocer si los pacientes entendían la información proporcionada.

Para preservar la confidencialidad de los datos se asignó a cada individuo un código único. Este código estaba ubicado en la carta de presentación (pre-test y posttest). La base de datos tenía una variable de identificación codificada de acceso restringido. El análisis estadístico se realizó sobre datos individuales carentes de información que permitiese la identificación de los participantes.

\section{ANÁLISIS ESTADÍSTICO}

Los datos categoriales se analizaron con la prueba de $\chi^{2}$, mientras que los datos cuantitativos se analizaron con las pruebas t o con análisis de varianza, utilizándose, en este último caso, la corrección de Bonferroni. En todos los casos se tomó como criterio de significación una prueba bilateral con valor $\mathrm{p}<0,05$.

\section{RESULTADOS}

En la Tabla 2 se indica el porcentaje de casos que, antes de llevar a cabo la primera visita, presentaban malestar emocional en alguno de los indicadores y para cada uno de los diagnósticos por los que acudían a la Unidad de consejo genético. La presencia de malestar emocional no guardaba relación con el diagnóstico, ni tampoco con el género, la edad, o el tener hijos. Sin embargo, la situación laboral sí guardaba relación con el indicador "Sentirse nervioso", puesto que hubo un mayor porcentaje de personas que mostraron 
Tabla 2. Relación entre malestar emocional y diagnóstico, antes de la primera visita

\begin{tabular}{|l|cc|cc|cc|}
\hline \multirow{2}{*}{$\mathrm{N}=43$} & \multicolumn{2}{|c|}{ Sentirse nervioso/a } & \multicolumn{2}{c|}{ Sentirse Triste } & \multicolumn{2}{c|}{ Sentirse preocupado/a } \\
& $\mathbf{n}(\mathbf{\%})$ & $\mathbf{n}(\mathbf{\%})$ & $\mathbf{n}(\mathbf{\%})$ & $\mathbf{n}(\mathbf{\%})$ & $\mathbf{n}(\mathbf{\%})$ & $\mathbf{n}(\%)$ \\
\cline { 2 - 7 } & $\mathrm{NO}$ & $\mathrm{Sí}$ & $\mathrm{NO}$ & $\mathrm{Sí}$ & $\mathrm{NO}$ & Sí \\
$\mathrm{CMOH}$ & $10(23,25)$ & $5(11,53)$ & $9(20,93)$ & $6(13,95)$ & $11(25,58)$ & $4(9,3)$ \\
$\mathrm{CCHNP}$ & $17(39,53)$ & $11(25,58)$ & $21(48,83)$ & $7(16,27)$ & $25(58,13)$ & $3(6,9)$ \\
Total & $27(63)$ & $16(37)$ & $30(70)$ & $13(30)$ & $36(84)$ & $7(16)$ \\
\hline
\end{tabular}

$\mathrm{CMOH}$ : Cáncer mama y/o ovario hereditario.

CCHNP: Cáncer de colon hereditario no polipósico.

\section{Tabla 3. Descripción del malestar emocional momentos después de haber recibido asesoramiento genético}

\begin{tabular}{|l|c|c|}
\hline ¿Cómo se siente usted en este momento? & $\mathbf{N}^{\circ}$ casos & Porcentaje \\
\hline No contesta & 5 & 9,3 \\
\hline Mucho más tranquilo que cuando Ilegué & 2 & 4,7 \\
\hline Bastante más tranquilo que cuando llegué & 5 & 11,6 \\
\hline Un poco más tranquilo que cuando llegué & 20 & 46,5 \\
\hline Confuso o aturdido & 9 & 20,9 \\
\hline Un poco más nervioso que cuando llegué & 2 & 4,7 \\
\hline Total & 43 & 97,7 \\
\hline
\end{tabular}

nerviosismo entre las que no realizaban actividad laboral (55\%) que entre las que sí la hacían $(21,7 \%)$, siendo esta diferencia estadísticamente significativa $\left(\chi^{2}=(1\right.$, $\mathrm{N}=43)=5,065, \mathrm{p}=.024)$.

Respecto al efecto inmediato sobre el estado emocional producido por la visita, la Tabla 3 indica que un $25 \%$ de los pacientes (11 casos) que contestaron a esta pregunta señalaron que la visita no les había tranquilizado, ya que dijeron sentirse confusos o aturdidos (9 casos) o un poco más nerviosos que antes de la visita (2 casos).

Los niveles de malestar emocional observados al cabo de 2-3 semanas después de la visita se indican en la Tabla 4. Globalmente, los datos indican un aumento del porcentaje de pacientes que se ubican en una situación de ausencia de malestar emocional, excepto en el ítem "sentirse preocupado".

La percepción de riesgo antes y después de la visita no guarda relación con el diagnóstico. La Tabla 5 muestra que hay diferencias que alcanzan la significación estadística $(F=33,73 ; p<, 001)$ en el riesgo percibido, antes de la visita, de padecer cáncer al cabo de un año, a 10 años, o a lo largo de toda la vida. El riesgo percibido a un año es inferior al percibido a los 10 años y a lo largo de toda la vida $y$, asimismo, el riesgo percibido a los 10 años es también inferior al riesgo percibido a lo largo de toda la vida. Dos o tres semanas después de la visita, estas diferencias cambian y solamente la percepción de riesgo a un año 


\section{Tabla 4. Malestar emocional antes y después (al cabo de 2-3 semanas) de la primera visita}

\begin{tabular}{|c|cc|cc|cc|}
\hline \multirow{2}{*}{ N=43 } & \multicolumn{2}{|c|}{ Sentirse nervioso/a } & \multicolumn{2}{c|}{ Sentirse Triste } & \multicolumn{2}{c|}{ Sentirse preocupado/a } \\
& $\mathbf{n}(\%)$ & $\mathbf{n}(\%)$ & $\mathbf{n}(\%)$ & $\mathbf{n}(\%)$ & $\mathbf{n}(\%)$ & $\mathbf{n}(\%)$ \\
\cline { 2 - 7 } & $\mathbf{N O}$ & Sí & NO & Sí & NO & Sí \\
Antes & $27(63)$ & $16(37)$ & $30(70)$ & $13(30)$ & $36(84)$ & $7(16)$ \\
Después & $31(72)$ & $12(28)$ & $36(84)$ & $7(16)$ & $34(79)$ & $9(21)$ \\
\hline
\end{tabular}

\section{Tabla 5. Percepción del riesgo antes y después (al cabo de 2-3 semanas) de la primera visita}

\begin{tabular}{|l|c|c|}
\hline & ANTES & DESPUÉS \\
& M (SD) & (SD) \\
\hline Al año & $4,60(3,57)$ & $4,31(3,28)$ \\
A los 10 años & $7,28(2,54)$ & $6,33(2,93)$ \\
A lo largo vida & $8,16(2,70)$ & $6,60(2,82)$ \\
\hline
\end{tabular}

sigue siendo inferior a la percepción de riesgo a 10 años o a lo largo de la vida $(\mathrm{F}=14,79 ; \mathrm{p}<, 001)$, ya que la reducción de la percepción de riesgo a lo largo de la vida, pasa de 8,16 a 6,60 , siendo esta disminución estadísticamente significativa $(t=3,099 ; p=, 003)$. La disminución de la preocupación a 10 años también disminuye, pero no alcanza la significación estadística $(t=1,47 ; p=, 08)$.

\section{DISCUSIÓN}

Los resultados de nuestro estudio indican que los pacientes que acuden por primera vez a la unidad de consejo genético con sospecha de $\mathrm{CMOH}$ y CCHNP, presentan niveles de malestar emocional en alrededor del $30 \%$ de los casos, si tenemos en cuenta las respuestas dadas a los ítems que evalúan nerviosismo o tristeza. Así pues, nuestros resultados son comparables a los que se observan tanto en el entorno cultural anglosajón ${ }^{(5)}$ como en el nuestro(4).
No obstante, debemos señalar que Cabrera y colaboradores ${ }^{(1)}$ no encontraron en su muestra de pacientes que acudían a la primera visita de consejo genético niveles de ansiedad que alcanzasen el grado de malestar emocional. Dado que estos datos provienen de una muestra española, no podemos atribuir esta discrepancia a diferencias culturales, por lo que quizá ésta se deba a la utilización de instrumentos de medida y de criterios para establecer el malestar emocional distinto a los de nuestro estudio. Por otra parte, el hecho de que el porcentaje de malestar registrado con el ítem preocupación fuera inferior puede explicarse en base a que no hay una causa concreta por la cual preocuparse, ya que la persona está asintomática, sin que ello impida que pueda haber nerviosismo o tristeza por el hecho de tener que acudir al hospital. Tras la visita, es posible que se tome conciencia de que existen motivos de preocupación y eso justificaría el aumento de casos con malestar emocional 
en este indicador que se observan al cabo de unas semanas.

Respecto a la posible relación entre malestar emocional y diagnóstico, los resultados de la literatura son escasos y contradictorios, ya que algunos trabajos señalan que las que tienen más malestar son las pacientes de $\mathrm{CMOH}^{(11)}$ y otros indican que son los pacientes de $\mathrm{CCHNP}^{(4)}$. En nuestro trabajo no hemos encontrado relación entre diagnóstico y malestar emocional, en la línea del trabajo de Van Oostrom y colaboradores $^{(9)}$. Es posible que realmente no existen diferencias o que el escaso número de casos de nuestra investigación no nos haya permitido detectarlas. A favor del primer supuesto, cabe argumentar que el desconocimiento en la sociedad española de lo que significa el consejo genético puede ser la razón que explique la ausencia de diferencias, ya que en el ámbito anglosajón, el mayor tiempo transcurrido desde la aplicación del consejo genético puede haber facilitado que la información que dispone la población permita que se generen unas expectativas diferentes y que éstas influyan en el malestar emocional previo al diagnóstico. Tampoco hemos observado diferencias en el malestar previo en función de otras variables como el nivel de estudios o el tener o no hijos. En cambio sí hemos hallado que el malestar emocional previo es mayor cuando la persona no realiza actividad laboral. No hemos encontrado resultados similares en la literatura, por lo que sólo podemos especular con que el no tener una actividad laboral que permita distraer a la personas de la inquietud de la proximidad de la visita, incremente el nivel de nerviosismo y ansiedad ocasionado por pensamientos negativos respecto a lo que la visita puede aportar.

Un resultado destacable de nuestro estudio es el que muestra que un $25 \%$ de los pacientes dicen que inmediatamente después de la visita de consejo genético se encuentran emocionalmente peor. No podemos cotejar este dato con la literatura porque, al igual que en el caso de la actividad laboral, no hemos encontrado trabajos que analicen este factor. En nuestra opinión, lo que puede estar ocurriendo en estos casos es que la persona acude a la visita sin tener una idea adecuada sobre el motivo de la misma, y al recibir en la visita de consejo genético una información que le hace ubicarse en la situación en la que realmente le corresponde, está reaccionando de la manera que cabe esperar en una coyuntura semejante, es decir, reaccionando con nerviosismo, tristeza o preocupación ante una mala noticia. Esta interpretación vendría avalada por los datos de nuestro estudio que muestran que al cabo de dos o tres semanas el malestar emocional se ha reducido, lo cual ha sido observado también en algunos trabajos que han evaluado el malestar emocional un mes después de la visita en el caso del cáncer de mama ${ }^{(12)}$ y al cabo de varios meses $^{(13)}$ en pacientes con diagnóstico de CCHNP. No obstante, hay que considerar que en el caso del cáncer de mama esta relación no es tan clara, pues se ha mostrado que los niveles de malestar previos a la visita pueden mantenerse durante bastante tiempo ${ }^{(14-15)}$.

La evaluación de la percepción del riesgo antes la visita indica que la preocupación al año es claramente inferior a la expresada al referirse al riesgo a 10 años o a lo largo de la vida, posiblemente porque las personas valoran que la probabilidad de tener cáncer a un año vista es muy baja. Globalmente estos resultados indican una sobreestimación del riesgo real, lo cual está de acuerdo con los resultados de otros trabajos ${ }^{(16-18)}$. Por lo tanto cabria esperar que una buena vista de consejo genético redujese los niveles de percepción del riesgo y los aproximase a los niveles de riesgo objetivo. Nuestros resultados indican que, después de la visita, 
efectivamente se reduce la percepción del riesgo a lo largo de la vida, pero que no varía el riesgo percibido a un año y a 10 años. Es difícil explicar porque la percepción del riesgo a un año y a 10 años no ha disminuido. Si los pacientes no hubieran entendido la información, su percepción de riesgo a lo largo de la vida no habría disminuido. Además, el trabajo de Sirgo y colaboradores, ${ }^{(19)}$ elaborado en un entorno cultural similar al nuestro, muestra que los pacientes adquieren una buena comprensión del nivel de riesgo que tienen. Sin embargo, podría ocurrir que al entender la persona que pertenece a un grupo que tiene mayor riesgo que la población general de padecer cáncer, asuma que este nivel de riesgo es constante en el tiempo, y por esta razón tiende a aproximar las tres valoraciones, ya que tras la visita los valores de riesgo a diez años se reducen, si bien no de forma que se alcance la significación estadística. No obstante no podemos estar seguros que esta sea la explicación. Hay estudios $^{(20)}$ que señalan que la percepción de riesgo se reduce en el caso de los no portadores y se mantiene entre los portadores, pero estas consideraciones no son aplicables al caso de nuestra investigación, ya que en la primera visita todavía no se conocía el resultado del test genético.

Nuestra investigación aporta datos que avalan la idea de que el malestar emocional es un elemento importante en el contexto de una primera visita de consejo genético y que dicha visita reduce, en parte, la sobreestimación de riesgo de padecer cáncer. No obstante, debe tenerse en cuenta que la muestra se compone de un número escaso de pacientes, en el que hay muy pocos hombres y en el que aparecen dos tipos de tumores, lo que disminuye aún más el número de casos valorados en cada categoría concreta. Debemos señalar que los estudios que se han venido publicando a partir del año 2003 en el ámbito anglosajón en su inmensa mayoría trabajan con más de 100 casos y que en estudios más recientes las cifras superan los 200. De todas formas los pocos estudios publicados en el ámbito español tienen muestras de tamaño similar a la nuestra ${ }^{(2,19)}$, aunque están empezando a aparecer trabajos con muestras más grandes ${ }^{(15)}$. En segundo lugar, el hecho de utilizar un envío por correo para la valoración a las 2-3 semanas hace que la muestra que se dispone sea escasa.

Al margen de esas consideraciones, creemos que ha quedado evidenciada la necesidad de que las unidades de consejo genético tengan en cuenta el papel del malestar emocional e incluyan en sus protocolos de visita estrategias que garanticen un buen manejo del mismo incorporando medidas específicas sobre la preocupación que genera el motivo de consulta ${ }^{(21)}$. Para ello pueden usarse intervenciones sencillas basadas en protocolos de información breves, recurso que ha sido ya probado de forma satisfactoria fuera de nuestro entorno cultural ${ }^{(22)}$.

\section{REFERENCIAS BIBLIOGRÁFICAS}

1. Cabrera E, Yagüe C, Schaiffino A, Llort G, Blanco I. Información, percepción de riesgo y ansiedad antes de recibir consejo genético en la predisposición hereditaria al cáncer. Enferm Clin 2006; 16(3): 112-8.

2. Gil F, Méndez I, Sirgo A, Llort G, Blanco I, Cortes-Funes, H. Perception of breast cancer risk and surveillance behaviors of women with family history of breast cancer: A brief report on a Spanish cohort. Psychooncology 2003; 128(8): 821-7. doi:10.1002/ pon.704

3. Coyne JC, Benazon NR, Gaba CG, Calzone K, Weber BL. Distress and psychiatric morbility among women from high-risk breast and ovarian cancer families. J Consult Clin Psychol 2000; 68(5): 864-874. Doi:10.1037//0022-006X.68.5.864

4. Cruzado JA, Pérez-Segura P, Olivera $H$, Sanz R, Hernández V, Suarez A. et al. Ne- 
cesidad de tratamiento psicológico en personas con riesgo de cáncer hereditario que inician consejo genético. Estudio de variables predictoras. Psicooncología 2005; 2(23):303-16.

5. Matthews A, Brandenburg D, Cummings S, y Olopade O. Incorporating a psychological counselor in a cancer risk assessment program: necessity, acceptability, and potencial roles. J Gen Couns 2002; 11(1): 5164. Doi:10.1023/A:1013820515603

6. Meiser B, Halliday JL. What is the impact of genetic counselling in women at increased risk of developing hereditary breast cancer? A meta-analytic review. Soc Sci Med 2002; 54(10):1463-7. Doi:10.1016/S02779536(01)00133-2

7. González M, Blanco I, Bayes R. Dificultad en la comunicación del riesgo de padecer cáncer hereditario. Psicooncología 2005; 2 (2-3):285-2.

8. Watson M, Foster C, Eeles R, Eccles D, Asheley S, Davidson R. et al. Psychosocial impact of breast/ovarian (BRCA1/2) cancer-predictive genetic testing in a UK multi-centre clinical cohort. $\mathrm{Br} J$ Cancer 2004; 91(10):1787-94. Doi:10.1038/ sj.bjc. 6602207

9. Van Oostrom I, Meijers-Heijboer H, Duivenvoorden $H J$, Bröcker-Vriends $A H$, van Asperen, CJ, Sijmons, RH, et al. Comparison of individuals opting for BRCA $1 / 2$ or HNPCC genetic susceptibility testing with regard to coping, illness perceptions, illness experiences, family system characteristics and hereditary cancer distress. Patient Educ Couns 2007; 65(1): 58-68. Doi:10.1016/j. pec.2006.05.006

10. Lipkus IM, Kuchibhatla M, McBride CM, Boswoeth HB, Pllak KI, Siegler IC, et al. Relationships among breast cancer perceived absolute risk, comparative risk, and worries. Cancer Epidemiol Biomarkers Prev 2000; 9(9): 973-5.

11. Wakefield CE, Meiser B, Homewood J, Barlow-Stewart K, Tucker, K.. A comparison of community, clinician, and patient preferences for naming a cancer-related mutation. Clin Gen 2007; 7(2):140-7. DOI: 10.1111/j.1399-0004.2007.00754.x

12. Foster C, Evans DG, Eels R, Eccles D, Ashley S, Brooks, L. et al. Non- uptake of predictive genetic testing for BRCA1/2 among relatives of know carries: attributes, cancer worry, and barriers to testing in multicenter clinical cohort. Gen Test 2004; 8(1):23-9. Doi:10.1089/109065704323016003

13. Shiloh S, Koehly L, Jenkins J, Martin J, Hadley D. Monitoring coping style moderates emotional reactions to genetic testing for hereditary nonpolyposis colorectal cancer: A longitudinal study. Psychooncology 2008; 17(8):746-55. Doi:10.1002/pon.1338

14. Smith AW, Liegey Dougall A, Posluszny D, Somers T, Rubinstein W, Baum A. Psychological distress and quality of life associated with genetic testing for breast cancer risk. Psychooncology 2008; 17(8):767-73. Doi:10.1002/pon.1291

15. Cabrera E, Blanco I, Yagüe C, Zabalegui A. The impact of genetic counselling on knowledge and emotional responses in Spanish population with family history of breast cancer. Patient Educ Couns 2010; 78(3):382-8. Doi:10.1016/j. pec.2009.10.032

16. Codori AM, Waldeck T, Petersen GM, Miglioretti D, Trimbath JD, Tillery MA. Genetic counseling outcomes: Perceived risk and distress after counseling for hereditary colorectal cancer. J Genet Couns 2005; 14(2):119-32. Doi:10.1007/s10897-005-4062-2

17. Evans DG, Hopwood P, Howell A. Perception of risk in women with a family history of breast cancer. Br J Cancer 1993; 67(3):612-4. Doi:10.1038/bjc.1993.112

18. Watson M, Lloyd S, Davidson J, Meyer L, Eeles $\mathrm{R}$, Ebbs, $\mathrm{S}$ et al. The impact of genetic counselling on risk perception and mental health in women with a family history of breast cancer. Br J Cancer 1999; 79 (5-6): 868-74. Doi:10.1038/sj.bjc.6690139

19. Sirgo A, Rubio B, Torres A, Salva M, Brunet J. Impacto psicosocial del test genético 
en pacientes diagnosticados con cáncer de mama o colorrectal y familias de alto riesgo: la atención psico-oncológica en el hospital universitario Sant Joan de Reus. Psicooncología 2005; 2(2-3): 369-82.

20. Wagner A, van Kessel I, Kriege, MG, Tops CM, Wijnen JT, Vasen HF, et al. Long term follow-up of HNPCC gene mutation carriers: compliance with screening and satisfaction with counseling and screening procedure. Fam Cancer 2005; 4(4):295-300. Doi:10.1007/s10689-005-0658-9
21. Cabrera E, Zabalagui A, Blanco I. Versión española de la Cancer Worry Scale (escala de preocupación por el cáncer: adaptación cultural y análisis de la validez y la fiabilidad). Med Clin 2010. Doi:10.1016/j.medcli.2010.04.015

22. Bennett C, Burton H, Farndon P. Competences, education and support for new roles in cancer genetics services: Outcomes from the cancer genetics pilot projects. Fam Cancer 2007; 6(2):171-80. Doi:10.1007/ s10689-007-9127-y 
